\title{
Escenarios de incertidumbre: crimen organizado trasnacional y potenciadores del riesgo ${\text { (Brasil y Chile })^{1}}^{1}$
}

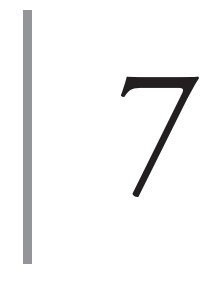

https://doi.org/10.21830/9789585241480.07

\author{
Sara Patricia Quintero Cordero ${ }^{2}$ \\ Escuela Militar de Cadetes "General José María Córdova” \\ Manuel Forero-Garzón ${ }^{3}$ \\ Universidad Uniminuto \\ Wilson Cardoso Ulloa ${ }^{4}$ \\ Centro de Misiones Internacionales y Acción Integral
}

\section{Resumen}

El presente capítulo precisa el análisis de las estrategias de defensa de Brasil y Chile ante el Crimen Organizado Transnacional (COT). Este estudio se fundamenta en la dinámica del Sistema Internacional, donde el Estado ya no es el único actor preponderante

1 Este capítulo hace parte de los resultados del proyecto de investigación titulado "La construcción de la identidad nacional en Colombia a partir de la consolidación de la seguridad nacional", del Grupo de Investigación en Ciencias Militares, de la Escuela Militar de Cadetes "General José María Córdova" (ESMIC), registrado con el código COL0082556 de Colciencias. Los puntos de vista y los resultados de este capítulo pertenecen al autor y no reflejan necesariamente los de la ESMIC o el Ejército Nacional de Colombia.

2 Internacionalista. Estudiante de pregrado en Derecho y estudiante del Doctorado en Derecho de la Universidad de Buenos Aires. Magíster en Relaciones Internacionales de la Universidad de Buenos Aires y Magíster en Seguridad y Defensa Nacionales de la Escuela Superior de Guerra "General Rafael Reyes Prieto". Docente e investigadora de la Facultad de Relaciones Internacionales de la Escuela Militar de Cadetes "General José María Córdova”. Orcid: https://orcid.org/0000-0002-9053-377X. Contacto: sara. quintero@esmic.edu.co

3 Profesional en Relaciones Internacionales y Estudios Políticos. Magíster en Relaciones Internacionales. Estudiante del Doctorado en Seguridad Internacional de la Universidad Nacional de Educación a Distancia (UNED). Profesor investigador de la Uniminuto Centro Regional Girardot. Contacto: mforero@uniminuto.edu

4 Coronel del Ejército Nacional de Colombia. Especialista en Seguridad y Defensa de la Escuela Superior de Guerra "General Rafael Reyes Prieto". Especialista en Administración de la Seguridad de la Universidad Militar Nueva Granada. Especialista en Administración y Gerencia Deportiva unidad del Centro de Educación Militar. Profesional en Ciencias Militares de la Escuela Militar de Cadetes "General José María Córdova”. Orcid: https://orcid.org/0000-0002-6937-8250. Contacto: wilson.cardoso@buzonejercito.mil.co 
y los temas de la Agenda de la Seguridad internacional han incorporado amenazas diferentes a las tradicionales, que afectan la seguridad de los Estados, elementos presentes en el referente teórico de la Escuela de Copenhague. Los dos Estados estudiados evidencian en sus políticas de seguridad y defensa nacional valores de desarrollo en relación con el interés nacional, al considerar que alcanzar niveles aceptables de desarrollo es esencial para combatir el COT. Las amenazas relacionadas con este crimen son afrontadas de forma multidimensional combinando factores sociales, políticos, económicos, culturales y militares que implican la funcionalidad del Estado y sus instituciones y la Cooperación Internacional.

Palabras claves: Brasil; Chile; crimen organizado transnacional; defensa y seguridad

\section{Introducción}

El fenómeno de la globalización ha conducido a discusiones desde perspectivas sociales, políticas, académicas y culturales de diferentes ciencias y disciplinas, que han analizado su efecto sobre el Estado en su organización y funcionalidad. Dentro de estas ciencias, particularmente las relaciones internacionales centran sus fines ontológicos y epistemológicos en la relación entre los diferentes actores del sistema internacional y cómo interactúan para lograr sus fines políticos. Inicialmente esta discusión resaltó que el Estado había sido desdibujado en su papel y estaba siendo presionado para reducir su influencia en la escena internacional, por lo tanto, se pronosticaba la desaparición de las fronteras. Pero la dinámica actual del sistema internacional (multipolarismo), que sin duda se desenvuelve dentro de la dinámica multidimensional de la globalización, ha logrado el efecto contrario, es decir, el Estado ha adquirido mayor relevancia y la seguridad internacional, con sus amenazas tradicionales y nuevas, es objeto de estudio y revisión desde las políticas públicas de los diferentes gobiernos por considerarla esencial para su estabilidad.

La evolución de las relaciones internacionales como disciplina científica condujo al desarrollo de paradigmas, enfoques o escuelas teóricas con la intención de crear un marco de referencia que intenta explicar lo que sucede en la realidad del escenario internacional. Dentro de esta discusión epistemológica, la seguridad internacional también adquirió especial atención al desarrollar corrientes en las cuales los escenarios y amenazas experimentan cambios para ajustarse a la actual dinámica del sistema internacional. 
Buzan et al. (1998), al abordar estudios sobre la agenda de la seguridad internacional, advierten que la evolución de esta precisa de tres estadios o etapas. La primera de ellas es un enfoque tradicional que asume que el factor militar y político son los instrumentos adecuados para la defensa y seguridad de los Estados. En la segunda etapa se integran otras dimensiones del poder que se apartan de las tradicionales y, en la última, los Estados ya no son los únicos actores preponderantes en el estudio, la dinámica y la aplicación de la seguridad. Los citados autores señalan tres orientaciones específicas de esta disciplina en las relaciones internacionales: en primer lugar, los tradicionalistas, que sostienen que la agenda de la seguridad internacional está marcada por los temas políticos y militares y, si existe la inclusión de otros, estos deben estar ligados íntimamente a los primeros, siendo una visión Estado-centrista. La segunda está a cargo de los críticos, quienes catalogan a los individuos como actores del sistema internacional, pero no con la misma proporción de decisión e influencia que las unidades y subsistemas en la agenda de la seguridad internacional. La tercera orientación, los ampliacionistas, bajo una óptica constructivista amplían la naturaleza (la militar no es la única) de las amenazas, que representan problemas con características sociales, económicas y ambientales, entre otras, y que requieren de políticas públicas asertivas para la movilización de la institucionalidad estatal para hacer frente a las amenazas contra la seguridad del Estado.

Continuando con Buzan et al., la seguridad internacional es extendida a los sectores económico (relaciones de comercio, finanzas y producción), social (relaciones de identidad colectiva) y ambiental (relaciones del ser humano con su entorno de biosfera). Es decir, ellos consideran que cuando un objeto está bajo amenaza se reacciona desde los diferentes sectores: el político, militar, económico, social y ambiental. Por ende, es el Estado a quien se le demanda la solución y la garantía de la seguridad, por ello es de vital importancia observar cómo desde otras lógicas teóricas de las relaciones internacionales, como la Escuela de Copenhague, el fortalecimiento de las agendas de seguridad interroga el papel discursivo gubernamental y, de esta manera, quieren evitar el escenario de incertidumbre que trae el crimen organizado transnacional —en adelante, COT_- De acuerdo con lo anterior, este capítulo abordará aspectos 
de las estrategias de defensa de los Estados de Brasil y Chile, en la lucha contra el crimen organizado y dos potenciadores del riesgo5: el narcotráfico y las migraciones.

\section{Potencia regional y entorno del COT}

En el estudio de las relaciones internacionales es posible establecer periodos históricos para abordar el análisis de realidades complejas que marcan el devenir del sistema internacional. Uno de ellos es la desintegración de la URSS y la finalización de la Guerra Fría, que coinciden con la mutación de la seguridad internacional abordada en los párrafos anteriores. Eric Hobsbawn (1998) retrata el siglo XX como un siglo corto, que inicia en 1914 con la Gran Guerra y finaliza con el fin de la Guerra Fría en 1991, etapa histórica que, según Villalpando (2014), coincide con el surgimiento del COT. Este último autor lo considera como una tercera etapa del crimen, que ya no presenta una estructura piramidal como la del crimen organizado, sino una red que conduce acciones ilegales que traspasan las fronteras con fines lucrativos valiéndose de la debilidad de los Estados y cuyos actores, en la mayoría de las ocasiones, no se conocen. Ante el avance del COT, la comunidad internacional, bajo el auspicio de las Naciones Unidas (ONU) y el Derecho internacional, a principios del siglo XXI, reaccionan con la Convención de las Naciones Unidas contra la Delincuencia Organizada Transnacional de $2002^{6}$.

En toda esta variación del sistema internacional —entendida como de la percepción de las amenazas a la seguridad, de la dinámica de la política internacional y de la variedad de actores que intervienen en esta interrelación de variables-, la geopolítica sigue siendo un instrumento eficaz para la planeación, diseño y ejecución de políticas internas y externas, orientadas a la

5 Hace referencia a "una serie de fenómenos de carácter global «que propician la propagación o transformación de las amenazas y riesgos” (García, 2013, p.146).

6 Aunque en el desarrollo de esta cumbre no se proporciona un concepto sobre el crimen organizado transnacional, sí se realiza una caracterización de la delincuencia organizada transnacional: intervienen dos o más personas con la asignación de tareas diferentes, actuando de forma continua, traspasando las fronteras de los Estados y generando violencia. Las actividades relacionadas con este tipo de organización son la trata de personas, el tráfico ilegal de migrantes y el tráfico de armas (sus componentes, sus piezas y municiones). 
seguridad de un Estado y su evolución hacia el desarrollo. Quintero y Forero (2018) analizan el factor geopolítico del Libro Blanco de Defensa de Brasil (Política de Defensa Nacional) de 2012, donde regiones de valor estratégico como la Amazonía, la Amazonía Azul, la Antártida, el litoral del Atlántico Sur y la proyección al océano Pacífico, al África, a Europa y al Mar Caribe merecen protección por los recursos estratégicos allí presentes, por la dinámica regional, continental y global, puesto que están expuestas a la interacción del COT, lo cual se traduce al lenguaje común como amenazas tradicionales y nuevas a la seguridad. Mariano Bartolomé considera que lo que se entiende por nuevas amenazas no es una expresión adecuada al considerar que las que se rotulan bajo este adjetivo no son nuevas en realidad (2006, p. 161) ${ }^{7}$.

El capítulo I del Libro Blanco de Defensa de Brasil (el Estado brasileño y la defensa nacional) es una conjunción de ventajas comparativas y competitivas, determinismo geográfico, perfil de la política exterior brasileña, dependencia del comercio marítimo, respeto a los principios generales del derecho internacional y defensa y seguridad del Estado de Brasil. El objetivo es conducir el Estado a potenciar la seguridad nacional en función del bienestar, protección y el interés de su sociedad, mediante la capacidad de disuasión y la cooperación internacional (Brasil, 2012, pp. 15-29). Para Reynolds (1977, pp. 45-59), la relación entre seguridad y desarrollo se obtienen del Estado, el bienestar de su población y la creación de valores para esa sociedad; él lo denomina el interés nacional. En esta misma vía, Esquivel (2015) propone que todo interés geopolítico debe estar subordinado al interés nacional de los Estados.

La disuasión y la cooperación internacionales son el resultado de la categorización de las amenazas que se hace desde la Política de Defensa Nacional de Brasil, y que afectan las condiciones sociales de desarrollo de una sociedad cada vez más interesada por temas de la seguridad y defensa nacional. Entre las amenazas se destaca el interés de otros Estados por la importancia geopolítica de Brasil (la respuesta es la disuasión) y otras que son consideradas actividades propias del COT: narcotráfico, piratería, tráfico de armas, municiones y explo-

7 Este autor considera que una amenaza nueva es la guerra informática. 
sivos, así como la afectación al medioambiente, que necesariamente requieren del accionar del Estado y de la cooperación de otros Estados.

En los escenarios continentales y regionales el COT es tema de estudio y de investigación por su impacto en la seguridad y afectación al desarrollo de los Estados. En 2003, durante el desarrollo de la Cumbre sobre Seguridad en las Américas, se estableció la visión multidimensional de las amenazas para la región. El Mercosur ${ }^{8}$ retoma la visión desarrollada en la OEA y prioriza las percepciones de las amenazas del área geográfica que representa esta organización internacional, siendo el orden el siguiente: narcotráfico, terrorismo, tráfico de armas, crimen organizado, medioambiente y desastres naturales, pobreza y carencias sociales, guerrillas y grupos subversivos (que se fortalecen y propagan por la interdependencia de los actores del sistema internacional y el constante desarrollo e innovación de las tecnologías de la comunicación y la información). Todo ello convierte al COT en una perfecta organización que pone a prueba la capacidad del Estado al debilitar su democracia e impactar el bienestar población y sociedad (Banegas, 2017, pp. 89-93).

Todo el potencial expuesto en el Libro Blanco de Defensa de Brasil confirma el papel de este país en la región como una potencia regional y con aspiraciones de incrementar su accionar y ser considerado un actor decisivo en el escenario global; pero esta demostración de poder e influencia no lo hacen inmune ante las amenazas del COT, que desdibuja las fronteras externas e internas. Si un Estado desea condiciones de seguridad, debe identificar plenamente esas amenazas que lo hacen percibir como inseguro.

Ullman, citado en Bartolomé (2016, p. 109), argumenta que una amenaza a la seguridad no es de naturaleza militar, sino más bien es la capacidad para impactar en un alto grado la calidad y dignidad de una población, reduciendo así las posibilidades políticas de los Estados. Brasil, al igual que América Latina, considera el COT como una amenaza que ha sobrepasado la capacidad de respuesta de los Estados, produciendo cambios en sus políticas públicas para contrarrestar los efectos del crimen en mención, por ejemplo, las nuevas funciones asignadas a las Fuerzas Armadas de ayuda a las fuerzas poli-

8 El Mercado Común del Sur es un modelo de integración que tuvo su origen en 1991, cuyos Estados parte son Brasil, Argentina, Paraguay, Uruguay y Venezuela. 
ciales y demás instituciones estatales que fueron superadas por las actividades propias del COT.

Bartolomé (2016), mediante una analogía de las amenazas transnacionales usada por Handley y Zieger, analiza el grado y percepción de peligrosidad en relación con el primer factor. Al combinar actores estatales y no estatales con peligro letal y no letal, el resultado son cuatro tipos de amenazas: I, Estados que persiguen sus objetivos con poder duro, y II, Estados con la suficiente capacidad letal para lograr sus objetivos (ambos catalogados como amenazas letales); y los tipos III y IV (dentro de los cuales se encuentra el crimen organizado), que están catalogados como no letales.

La percepción de peligrosidad establece las amenazas en niveles de inminente y largo plazo. En referencia al primer nivel, la peligrosidad se considera algo constante a nivel global, pero con implicaciones al interior de los Estados, por lo que requiere de una respuesta inmediata. El narcotráfico, el crimen organizado y la proliferación de armas convencionales son ejemplos de este tipo de amenaza. En el segundo nivel, los efectos nocivos son de menor impacto en comparación con las amenazas inminentes, lo que requiere de una menor asignación de recursos por parte del Estado para su neutralización, por ejemplo, el flujo migratorio masivo, la proliferación de armas de destrucción masiva $(\mathrm{ADM})$, el deterioro del medioambiente y la guerra informática (Bartolomé, 2016, pp. 159-163). Obsérvese que los ejemplos citados en el grado y la precepción de peligrosidad están relacionados con la dinámica del COT, exceptuando a las ADM.

Aunque la mención epistemológica de las amenazas transnacionales es rígida, no es necesaria su aplicación al pie de la letra. Bartolomé sostiene que cada Estado está en la capacidad de evaluar el impacto de estas amenazas de acuerdo con sus capacidades y cooperación con otros Estados, para finalmente decidir la gravedad y nivel de peligrosidad de estas y su posible respuesta (2016, p. 162). En una lógica proporcional, si Brasil categoriza las amenazas a su seguridad y defensa nacional empleando una escala de grado y percepción de peligrosidad, también afectará los intereses de este Estado. Donald Nuechterlein (1975) analiza cómo los intereses nacionales ${ }^{9}$ de los Estados

9 El autor en mención define, caracteriza y ejemplifica el interés público (bienestar del pueblo y empresas norteamericanas dentro de sus fronteras), el interés nacional (bienestar de los ciudadanos y empresas norteamericanos que participan en las relaciones internacionales), el interés estratégico (peligros 
varían en un mundo cambiante. Aunque su argumentación se focaliza en los intereses que han experimentado los Estados Unidos de América durante su evolución histórica, también realza analogías con otros Estados, siendo pertinente para el tema del presente capítulo de Brasil y el COT.

Para Brasil, el narcotráfico, la trata de personas, el flujo migratorio, tráfico de armas, explosivos y municiones, el contrabando, la minería ilegal, la corrupción, entre otras, son amenazas para su seguridad y defensa nacionales. Estas afectan el interés público por el accionar de las redes del COT que emplean la infraestructura del Estado, el no control efectivo del territorio, la importancia del determinismo geopolítico, la falta de ética de funcionarios estatales, la desigualdad y justicia social y la ineducada respuesta del Estado. Esto conduce finalmente a socavar el ejercicio de la democracia, el bienestar de la población brasileña, la economía, el control de la soberanía y la política de fronteras.

De igual forma, estas actividades ilícitas exigen desde el interés vital una respuesta unificada de Brasil en cooperación con la comunidad internacional. Nuechterlein considera este interés como aquellas posibles amenazas que afectan la supervivencia política y el bienestar económico del Estado, en donde el tiempo de respuesta requiere planeación para elaborar una escala de prioridades y no generar una respuesta inmediata que al mediano y largo plazo no solucionen esta problemática (1975, pp. 13-15). Buzan et al., por su parte, asumen las amenazas desde los sectores militar y político (cuando su política de defensa hace énfasis en el desarrollo de capacidades para lograr una disuasión) y de los sectores económico, social y ambiental (interdependencia, multilateralismo, cooperación internacional y reforzamiento del Estado brasileño).

\section{La potencia regional y su respuesta al COT}

La relación proporcional de la amenaza sobre la seguridad implica que si el Estado y sus poderes nacionales no reaccionan, los demás temas de la agenda

militares), el interés privado (actividades de ciudadanos y empresas norteamericanos en el extranjero), los intereses nacionales básicos (defensa, tráfico y comercio, ideología y creación de un orden mundial estable) y los intereses transitorios (capacidad y tiempo de reacción). Estos últimos el autor los subdivide en cuatro categorías: de supervivencia, vitales, principales y periféricos (Nuechterlein, 1975, pp. 1-36) 
estatal no podrán ser abordados y desarrollados. Desde la perspectiva de la corriente ampliacionista, propuesta por Buzan et al., se acepta que las amenazas sobre la soberanía cada vez son más difusas, por lo tanto, las Fuerzas Armadas, para enfrentar estas amenazas multidimensionales, implementan una reforma para el aumento de capacidades en la inteligencia operativa, que impera una ventaja cibernética, un Sistema Integrado de Monitoreo de Fronteras (Sisfron) cubriendo una área estratégica para la Escuela Geopolítica Brasileña como lo es la Amazonía.

Otro tema que reafirma esta corriente es la incorporación de la academia al tema de la seguridad y defensa nacionales. Se modificaron los planes de estudio de las facultades de relaciones internacionales y ciencias políticas, creando nuevos cursos con orientación a la defensa para su incorporación en procesos de investigación y abordar, así, todas las problemáticas que se generan en el escenario del COT. Toda esta dinámica es reunida en el Plan de Articulación de Equipamiento de Defensa (PAED), cuyo fin es el combate de las nuevas amenazas del crimen organizado y la salvaguarda de la sociedad brasileña en su crecimiento y desarrollo social (Brasil, 2012, p. 201).

De igual manera, la Política de Defensa de Brasil considera que sus Fuerzas Armadas son esenciales para garantizar la ley y el orden constitucional. Ejemplo de esto es la asignación de tareas nuevas a las Fuerzas Armadas para combatir las consecuencias del COT. Caso especial es la Fuerza Pacificadora, heredada de la experiencia de Brasil en la participación de las fuerzas de Asistencia Humanitaria de la ONU en Haití y creada en 2010 con la misión de establecer la paz social en las favelas de la periferia de Río de Janeiro. Este cambio de roles se efectuó legalmente mediante el Decreto N. 7.496 del 11 de junio de 2011 (neutralizar el crimen organizado) y faculta a las Fuerzas Armadas para combatir los delitos transfronterizos y ambientales en estados ${ }^{10}$ cuyos territorios son escenarios del COT (Brasil, 2012, p. 167).

Sampo y Alda (2019) explican cómo se desdibuja con funciones secundarias la misión principal de las Fuerzas Armadas, que es la defensa nacional. Lo explican en razón a que los Estados y, en especial, los de América Latina

10 Amazonas, Rio Grande do Sul, Paraná, Santa Catarina, Mato Grosso do Sul, Mato Grosso, Rondônia, Acre, Amapá, Pará y Roraima. 
son presionados por el nivel de inseguridad percibida en sus sociedades ante el avance del COT. Esto genera un nuevo escenario de seguridad donde las Fuerzas Armadas, bajo el rótulo de Fueras Multipropósito o Multimisión, se vuelcan a los escenarios de la seguridad interior, con lo cual desplazan las fuerzas policiales, que son incapaces de hacer frente a estas amenazas que ponen en riesgo al Estado y a su población.

Estas autoras, en su análisis de este escenario de seguridad para Latinoamérica, consideran que estas Fuerzas Multipropósito requieren de un cambio de doctrina, de capacitación en derechos humanos y derecho internacional humanitario, así como un marco jurídico regulatorio para cumplir la nueva misión encomendada. Ahora bien, si esto no sucede, se puede desembocar en una desprofesionalización de las Fuerzas Armadas. Continuando con esta parte del subcontinente, Sampo y Alda sostienen que estas fuerzas actúan con la estructura, material, equipo y doctrina ya existente, sin llevar a cabo modificaciones en la adaptación, modernización y transformación de las Fuerzas Armadas. Finalmente, realizan un análisis sobre cómo Brasil es un ejemplo de este cambio, al emplear a sus fuerzas en el mantenimiento del orden público, protección de infraestructura estratégica, control fronterizo, lucha contra el narcotráfico, combate a la extracción ilícita de recursos naturales (que son a la vez estratégicos) y tráfico de armas, explosivos y municiones.

Anais Medeiros (2019, p. 209) expone instrumentos jurídicos que amplían las funciones de las Fuerzas Armadas de Brasil para contrarrestar y neutralizar el crimen organizado, entre ellos, el Decreto N.o 3891, que establece funciones policiales para las Fuerzas Armadas; el Manual de la Garantía de la Ley y el Orden de 2014, que expande el apoyo de estas Fuerzas hacia las autoridades civiles; el Curso de Garantía de la Ley y el Orden para la Escuela de Comando Mayor y la Ley Complementaria N.o 97, que facultan a las Fuerzas Armadas para entrar en acción cuando las fuerzas policiales no cuentan con la suficiente capacidad de respuesta.

Buzan, citado por Sisco y Chacón (2004, p. 134), propone cinco niveles de análisis para el estudio de la seguridad: el sistema internacional (relaciones ámbito global), los subsistemas (organizaciones internacionales), las unidades (los Estados), las subunidades (grupos dentro de una unidad) y los individuos. 
Todos los niveles, después del dos, coinciden con los sujetos del derecho internacional, que poseen derechos y deberes ante la normatividad internacional, y que si llegasen a cometer delitos y crímenes internacionales serían puestos bajo esta jurisdicción.

Siendo Brasil una unidad con una importancia y proyección geopolítica destacadas en escenarios regionales, continentales y globales - que no pasa desapercibida por las redes del COT para lograr sus fines económicos-, firma en el ańo 2000 la Convención de la ONU Contra el Delito Organizado Transnacional y es ratificada en 2004. Los temas tenidos en cuenta por dicha convención incluyen los delitos de trata de personas, tráfico ilícito de migrantes, fabricación y tráfico de armas, narcotráfico, lavado de activos, extracción ilegal de recursos estratégicos y corrupción. En áreas de valor geopolítico para Brasil, como la Amazonía y la triple frontera, estas problemáticas son vivo reflejo de la realidad del COT y su impacto en la seguridad y defensa de este Estado.

Por su parte, las cuencas hidrográficas del Orinoco, la Amazonía y el Río de la Plata son una plataforma de infraestructura y red fluvial para el transporte y comercialización de bienes y servicios ilegales: minería ilegal, tala y tráfico de madera, comercialización de especies silvestres y narcotráfico. Por las condiciones geopolíticas de estas zonas, se encuentra salida hacia los mercados de Europa, África y, en menor medida, Asia. Brasil, que comparte frontera con tres unidades o Estados productores de cocaína (Colombia, Perú y Bolivia), y además, comparte frontera con Paraguay que es un gran productor de Marihuana, entiende que enfrentar el COT requiere de cooperación internacional, por lo cual adelanta operaciones conjuntas con estas unidades. Ejemplos de esta cooperación son las operaciones Ágata y Candado adelantadas con Colombia.

La Triple Frontera es una intersección entre Argentina (provincia de Misiones), Paraguay (departamento de Alto Paraná) y Brasil (Estado de Paraná), con obras de infraestructura que interconectan los tres Estados, además, en esta región geográfica se encuentra ubicado el Acuífero Guaraní, que es una de las mayores reservas de agua dulce subterránea del mundo. Paraná hace parte de la Región Sur, la cual es la menor en extensión territorial, densamente poblada y muy urbanizada, situación propicia para las actividades ilegales del 
COT. Cuervo-Ceballos (2018, p. 51) considera el crimen organizado como una verdadera amenaza a la gobernanza de Brasil, al hacer presencia allí redes criminales de origen chino, mexicano, colombiano, nigeriano, ruso y japonés, todos ellos, grupos especializados en la comercialización y contrabando de bienes electrónicos, tráfico de drogas, trata de personas y transferencias de dinero.

La diplomacia brasilera presente en Subsistemas como la ONU, la OEA, el Mercosur y Unasur ${ }^{11}$, refuerza la visión de que, en el nuevo escenario de la seguridad internacional, confluyen amenazas de diferente índole con la capacidad de afectar la estabilidad social, política, cultural, económica y militar de los Estados. Una realidad de la política internacional a nivel del continente americano ha sido su poca efectividad en el grado de integración y homogeneidad en enfrentar las amenazas, pero todas estas iniciativas pueden ser un punto de partida para lograr una multilateralidad en relación con los temas que afectan la seguridad y defensa de los Estados americanos.

\section{Chile: seguridad y COT}

Chile, en los últimos periodos presidenciales, ha afrontado y desarrollado de manera constante las estrategias para la implementación de las políticas de seguridad, con el objetivo de mejorar social y económicamente al país, construyendo nuevas pautas para la lucha dinámica y confiable contra el COT. Sin lugar a dudas, la globalización, los cambios constantes en otras políticas de seguridad de Latinoamérica y el mundo dificultan la evolución constante de la política pública en transición actual que lleva Chile. Con un gobierno constitucional duradero y estable posterior a la independencia, Chile se desatacó frente a otras repúblicas de América Latina por haber sido capaz de construir un Estado fuerte y unitario (Bethell, 2009). En este contexto, la política de seguridad es definida como

11 Unasur es una organización internacional de carácter regional que en sus inicios (2008) comprendía a doce Estados de Suramérica y que se ha debilitado políticamente por el retiro de Argentina, Brasil, Chile, Colombia, Perú y Paraguay. En la actualidad se pretende reemplazar esta organización por otra también de carácter regional, el Prosur, que muy seguramente terminará siendo una más. 
Las acciones que nacen del contexto social, pero que pasan por la esfera estatal como una decisión de intervención pública en una realidad social, ya sea para hacer inversiones o para una mera regulación administrativa. Se entiende por políticas públicas el resultado de la dinámica del juego de fuerzas que se establece en el ámbito de las relaciones de poder, relaciones esas constituidas por los grupos económicos y políticos, clases sociales y demás organizaciones de la sociedad civil. (Boneti, 2017, pp. 1-60)

Ahora bien, Chile posee fisuras en cuanto a la constancia y cubrimiento de pautas de políticas de seguridad de las Fuerzas Armadas, y no es una problemática actual, viene de atrás; sin embargo, en el país se hace necesario tener especial cuidado con la integración social y la seguridad, pues estos dos ítems vienen afectando de manera drástica el país: la doctrina de seguridad nacional (DSN) es una perspectiva académica que entendió la seguridad nacional en función de la defensa de los Estados frente a las influencias del comunismo, lo que llevó a generar tácticas militares de contención y disuasión (Ministerio de la Defensa Nacional de Chile, 2010). Las circunstancias del crimen organizado en Chile obligan al Estado a dar solución a aquellas problemáticas que las fuerzas militares consideran importantes. Es de anotar que cada una de ellas posee características importantes y transcendentes que, sin lugar a duda, permiten entender mejor las políticas públicas.

Las teorías son variadas en cuanto a causas y efectos, y ofrecen resultados diversos para cada país. Para los neoclásicos, que son quienes han hecho mayor desarrollo empírico, en el origen de las políticas públicas predominan las razones económicas por encima de otras razones. Sobre los efectos se viene promoviendo el análisis microterritorial debido a que no existe una distribución aleatoria, sino que se concentra en mayor proporción en las zonas de mayor crecimiento económico dentro del país de destino. (Gómez, 2010, pp. 81-99)

La política de seguridad pública de Chile está directamente relacionada con el Plan Nacional de Seguridad Pública y Prevención de la Violencia y el Delito 2014-2018, donde se estipulan los principales factores de diagnóstico encontrados por el Estado como de alta complejidad y pronta respuesta por parte de las Fuerzas Armadas chilenas ${ }^{12}$. En Chile, los homicidios están por

12 Cabe anotar que, la evolución de la seguridad desde la década de 1990 obtuvo un nuevo enfoque de seguridad, sin embargo, como se afirma a continuación: "Si bien la publicación del Informe de 
debajo de países como Colombia, Brasil y EE. UU., sin embargo, delitos como el narcotráfico, robos frustrados, abusos sexuales y la migración han subido dos dígitos cada año: "la percepción de inseguridad expresada por la ciudadanía, llevaron [sic] al gobierno chileno a buscar implementar acciones para hacer frente a tales situaciones y responder a la demanda ciudadana" (Ministerio del Interior y Seguridad Pública, 2014). Según Marín,

La política seguridad pública en Chile, comprendida para los años 2014 2018 responde a dos aspectos importantes. El primero de ellos a una preocupación instalada en la sociedad chilena por el tema de la delincuencia, siendo esta una de las principales prioridades en la agenda ciudadana y que termina por generar una sensación de inseguridad y temor al crimen o simple delito en los sectores tanto populares como en los sectores medios. (2016, p. 3)

El crimen organizado ha dejado una huella que perdura en el tiempo en países como Colombia y Chile, afectados por los altos índices de delincuencia común ocasionados por las represiones sociales, la falta de educación, las maniobras equivocadas del Estado y, por supuesto, la falta de políticas públicas que cubran los vacíos legales y judiciales de la democracia que dan lugar a omisiones en la represión de delitos. Sin embargo, cada país hace su mejor esfuerzo por combatir con sus fuerzas militares aquellas problemáticas, que están acompañadas del narcotráfico y, ahora en especial en Colombia, de la migración de ciudadanos venezolanos en condiciones de precariedad, que han aumentado las tasas de crimen organizado en toda Latinoamérica.

Por ello, la coordinación parece ser un esfuerzo importante en términos de generar soluciones a problemas prioritarios en materia de seguridad. De hecho, la coordinación fue uno de los pilares principales del Plan Nacional de Seguridad Pública y Prevención de la Violencia y el Delito, pilar que tenía como intención la optimización de recursos, una mayor efectividad de las medidas

Desarrollo Humano estableció la creciente interdependencia y la complejidad de las amenazas a seguridad, los atentados terroristas del 9-11 en Estados Unidos abrieron nuevamente el debate hacia el retorno del Estado como el garante de la seguridad internacional". (Quintero y González. 2017. p. 450) Y asimismo de la seguridad interior, se evidencia que las amenazas de diverso tipo siguen siendo soportadas por el Estado y principalmente por sus fuerzas militares y no por otros actores tales como organismos internacionales, o no gubernamentales. 
implementadas y la generación de metas y objetivos compartidos (Ministerio del Interior y Seguridad Pública, 2014). Así, resulta eminentemente necesario

El diseño e implementación de una profunda reforma de las estructuras orgánico-funcionales y doctrinales de las instituciones militares, con el fin de eliminar las profundas deformaciones que poseían; así como la revisión de las violaciones a los derechos humanos cometidas bajo el régimen militar, demanda que se sustentaba sobre un amplio consenso social. (Saín, 2002, p. 2)

Los derechos humanos también juegan un papel importante, dado que constituyen un punto frágil en cuanto a la creación y mantenimiento de las políticas públicas en Chile, con el fin de mantener la ciudadanía bajo control y a su vez garantizar los derechos humanos de quienes alteran el orden público, y es allí donde las fuerzas militares no solo deben respetar la ley sino la integridad humana de cada individuo. En el caso de las migraciones, los países procuran mantener a los extranjeros con las premisas mínimas de un digno vivir, y una de las maneras para permitirse esto es con una economía estable. El ICESCR (International Covenant on Economic, Social and Cultural Rights) ${ }^{13}$ contiene una excepción expresa en el artículo 2.3, en el que se afirma que "los países en desarrollo, teniendo debidamente en cuenta los derechos humanos y su economía nacional, podrán determinar en qué medida garantizarán los derechos económicos reconocidos en el presente Pacto a personas que no sean nacionales suyos" (Asamblea General de las Naciones Unidas, 1976).

La política migratoria chilena — como todos los Estados en el mundo sin excepción alguna-, en el marco de sus políticas de seguridad y nacionalismo, reconoce al extranjero como inmerso en la dialéctica de la negación del otro, del otro como inferior. Según Mármora, "estas identidades colectivas se configuran a través del principio de alteridad, el nosotros, que se construye a partir de la experiencia, real o simbólica, de ese otro. Es así que una sociedad construye simbólicamente y materialmente a ese otro” (Mármora, 1987, p. 7).

13 Pacto Internacional de Derechos Económicos, Sociales y Culturales. Aprobada y abierta a la firma, ratificación y adhesión por la resolución 2200A (XXI) de la Asamblea General de 16 de diciembre de 1966, entrada en vigor el 3 de enero de 1976, de conformidad con el artículo 2. 
En el contexto latinoamericano, especialmente en los países del Cono Sur, las políticas públicas en las últimas décadas han aumentado considerablemente. En el marco de los países del Cono Sur, Chile en los últimos 25 años ha experimentado un aumento sostenido de los flujos migratorios, drogas y prostitución, entre otras problemáticas sociales de gran envergadura. Esto se explica por los procesos de integración regional a los que se ha sumado Chile (Mercosur, Comunidad Andina de Naciones, Alianza del Pacífico, etc.) "han proyectado a este país a tener múltiples factores de estabilidad de orden económico, social, político y de seguridad" (Olea, 2013). En este contexto,

La Política planteada desde el Libro Blanco del Perú y Brasil como una herramienta útil para extraer elementos de diálogos que permitan un trabajo regional frente a las nuevas amenazas en las sociedades políticas de sus vecinos y en particular Colombia, por lo tanto, cabe destacar, que este análisis tendrá un pequeño acercamiento a lo que es Perú y Brasil como Estado en su organización administrativa y militar mostrando desde su política de seguridad y defensa las orientaciones mismas en su ejercicio de poder. (Quintero y Forero, 2018, pp. 89-117)

El Estado chileno, en pro de mitigar las problemáticas causadas por agentes del crimen organizado, trabaja día a día en promover y reorientar las políticas existentes basadas en los derechos humanos, así como las diferentes normativas que convengan para contrarrestar los delitos en el país, sin embargo, no es suficiente.

Como parte de su cultura estratégica, Chile ha trabajado desde antes de la transición a la democracia en el desarrollo de una comunidad de defensa, como un grupo constituido por militares y por civiles, provenientes principalmente de centros académicos y partidos políticos, cuyo común denominador es su conocimiento y experiencia en materias de Defensa y que tiene por propósito fundir las vertientes de pensamiento civil y militar en la investigación y discusión de los temas generales que tienen elación con la Defensa Nacional. (Libro Blanco de Defensa de Chile, 2010, p. 78)

Sin lugar a duda, la desigualdad que se vive en el país, la falta de empleo y los estándares bajos en la educación crecen cada día más, junto con la delincuencia común. Por otra parte, el nivel de vida de la población ha disminuido en los últimos diez años, generando así consecuencias irreversibles para el Estado y, por supuesto, para las fuerzas militares, dado que el pie de fuerza no 
cubre la demanda delincuencial que se encuentra en el país en la actualidad. En efecto,

El aumento del bienestar socioeconómico (es decir, del nivel de vida de la población) en los países ricos implica una enorme carga económica en los países más pobres porque son aquellos los que se benefician más del crecimiento del comercio mundial y del abaratamiento relativo de los productos agrícolas, y ahora industriales, de los países más pobres. (Casassas, 2005)

Las Fuerzas Militares de Chile han determinado factores importantes de análisis como es la aparición de nuevas estrategias para combatir el crimen organizado trasnacional, con la cooperación de otros gobiernos que tienen la misma finalidad, disminuir el impacto negativo de los agentes delictivos y disminuir, en consecuencia, problemáticas alternas como el déficit de salud y de educación. No obstante,

Las fuerzas militares y de policía deben fundarse en un modelo democrático, que garantice la seguridad pública, refrene la criminalidad desde la prevención del delito y no desde la persecución de los criminales y construya confianza en la ciudadanía hacia la institución policial y militar. (Quintero, 2020, p.6)

En esta medida,

La seguridad y defensa también constituyen un insumo decisivo en la economía, por tal razón muchos de los proyectos de desarrollo tecnológico de cada una de sus fuerzas cuentan con el respaldo científico de las universidades y, en general, de la academia. Conscientes de los errores del pasado, la estructura de seguridad y defensa en Chile es el resultado del trabajo coordinado de todos los actores de la sociedad, además, la importancia de generar espacios de consentimiento con otros actores del sistema internacional. (Marín, 2016)

La seguridad representa un alto impacto económico para un país, y el combatir el crimen organizado aún más. Chile no es la excepción, por eso debe generar espacios de reflexión en concordancia con los aspectos del pasado que han permitido el incremento de delitos y su confrontación, sin embargo, en problemáticas como la inmigración es imposible detener una oleada de agentes delictivos que provoquen que estas cifras asciendan cada año. ${ }^{14}$

14 En los países en vías de desarrollo y que hacen parte de diferentes tipos de integración ya sean económica y/o política, como es el caso de Chile, es interesante observar que, en materia de seguridad ciudadana, los resultados parecen atrasados comparados con políticas de seguridad y defensa nacional. 


\section{Potenciadores del riesgo}

\section{El narcotráfico}

En Chile el narcotráfico ha aumentado en un $18 \%$ en los últimos 10 años, lo que ha llevado a la trasformación de las políticas de seguridad de Colombia con países centroamericanos y EE. UU., dado que trabajan en conjunto para disminuir este flagelo. Por lo anterior,

en función del interés nacional, aspectos cotidianos de la vida social, como las interacciones sociales, el papel de los medios de comunicación, los procesos de cooperación institucional, entre otros aspectos del ámbito social, se pueden convertir en amenazas a la seguridad nacional del Estado, desde un punto de vista "Estadocéntrico". (Ayoob, 1995)

De tal manera,

No se puede lograr una solución a un problema multilateral con medidas unilaterales, no existe la posibilidad de que eso ocurra. No se acaba un problema que cobija muchos países y que implica muchos aspectos, solamente atacando uno de esos aspectos en uno de los países. En el mejor de los casos, existen unas estadísticas que muestran que se ha reducido el número de usuarios de la cocaína. Pero también se ha demostrado, que entre los usuarios el consumo es cada vez más frecuente y, en algunos casos, de drogas más peligrosas. (...) Estamos convencidos que las políticas contra el consumo, el lavado de dólares, el tráfico de insumos y la producción, requieren de una concertación y de un entendimiento especial. (Pardo, 1990, p. 7)

La lucha con el narcotráfico debe realizarse mediante alianzas entre los países involucrados. Por ello, el gobierno trabaja en lucha contra el crimen organizado trasnacional para combatir poco a poco el narcotráfico y esto ha dado resultado: en los últimos cuatro ańos se ha disminuido un 3,5\% este flagelo respecto a años anteriores.

De lo anterior, tiene diferentes aristas a analizar como por ejemplo "Uno de los factores que incrementan esta sensación es la geopolítica moderna de la región, que determina que las problemáticas de seguridad son elementos que solo se pueden resolver a escala doméstica. Un segundo factor es que no hay un país en la región que asuma el liderazgo e impulse del desarrollo de estrategias efectivas. Y un tercero es que los temas de seguridad pública generalmente se han asociado con el narcotráfico y la inmigración, sin tener en cuenta las particularidades, necesidades y contextos específicos de cada país" (González y Quintero, 2018). De esta manera frente a temas de migración y delitos son los Estados que internamente imponen e implementan estrategias, y aunque sean amenazas comunes para otros Estados vecinos y regionales. 


\section{La migración}

La migración es un tema de suma importancia para todos los países, dado que son muchas las personas que deciden dejar su lugar de residencia y trasladarse a otras naciones, con el fin de mejorar su calidad de vida, buscar nuevas oportunidades o escapar de problemáticas sociales que impiden su bienestar. Debido a esto, se genera la decisión de comenzar en otras latitudes y la migración se convierte en una solución, una alternativa e incluso una nueva etapa, pero esta situación no afecta solo a las personas que se movilizan, sino también a la sociedad en general, incluido el Estado.

La migración en Latinoamérica tiene mucho que ver con la economía del continente, dado que gran cantidad de las personas que emigran lo hacen por aspectos económicos, en consecuencia, lo que se observa es la disminución de la demanda interna a causa del encarecimiento del crédito externo. Además, hay contracción de las exportaciones provenientes de países asiáticos, lo cual afecta el precio de los materiales de América Latina, el crecimiento de los países desarrollados en campos como el turismo y el poco empleo. De la misma manera, crece el flujo de capitales, que se realiza de manera constante y a corto plazo, lo que ha repercutido sobre presiones inflacionarias y la depreciación de las monedas, obligando a las personas a emigrar a otros países.

La migración en Chile es uno de los puntos frágiles en la lucha contra el crimen trasnacional, dado que los inmigrantes en la actualidad poseen una participación en el 35,3\% de los crímenes como narcotráfico y tráfico de armas, y en dificultades sociales como la prostitución y mendicidad, generando una necesidad de una política de seguridad eficiente que permita disminuir estas situaciones. En efecto, parte de las implicaciones migratorias en los países del mundo entero son los determinantes normativos para la culminación de políticas de seguridad, por lo cual, el derecho internacional migratorio juega un papel importante entre el Estado y los ciudadanos. Chile, en pro de disminuir el crimen organizado, enfatiza en proyectos de manejo adecuado de problemáticas como el narcotráfico y la prostitución. No obstante, debido a las crisis migratorias, los Estados se obligan a crear políticas y garantías para la migración. 
Las crisis tienen considerables efectos a largo plazo en los migrantes y la sociedad. Por ello, la acción concertada de la comunidad internacional es primordial a efectos de: prevenir y prepararse para las situaciones de crisis; apoyar a los migrantes, los desplazados y las comunidades afectadas por situaciones de crisis, de conformidad con los principios humanitarios; y promover soluciones duraderas que pongan un término al desplazamiento. La labor para encarar las causas originarias de las crisis y los movimientos de población conexos debe formar parte de enfoques a más largo plazo con miras a la recuperación, la transición y el desarrollo sostenible. Más aún, la comunidad internacional debería responder a las situaciones de crisis en el entendido de que la migración es una consecuencia inevitable, y de que los empeños de recuperación y transición exigen que se tengan en cuenta las necesidades de los migrantes y de sus comunidades. (Organización Internacional para las Migraciones, 2017)

Por esto, al abordar las obligaciones de los Estados en el ámbito de las migraciones internacionales, según Betts (2011), el gobierno cobra varias formas, entre ellas, las políticas migratorias y los programas de cada país, debates y acuerdos entre Estados, foros multilaterales y procesos consultivos, actividades de organizaciones internacionales, al igual que las leyes y normas que permitan garantizar y proteger a los no nacionales. Todo ello, con el objeto de abordar de manera efectiva las particularidades de vulnerabilidad que se presentan en los territorios y en las fronteras. En efecto,

La clave de la cuestión reside en encontrar la causa que genera la repetición en el tiempo y en países distintos de esos procesos extraordinarios de regularización. Así en lugar de optar por actuar sobre los síntomas externos del fenómeno migratorio, como ocurre cuando se recurre a los procesos coyunturales de regularización, podrá conseguirse su tratamiento global. (De Lucas, 1999, p. 1)

En este orden, Chile posee actualmente interrogantes importantes sobre la migración internacional, dado el alto nivel de esta afectación. La entrada y salida de migrantes afecta sus economías, las tasas de empleo y seguridad. Sin lugar a dudas, cada una de estas naciones propicia políticas y acuerdos bilaterales que les permitan avanzar en un derecho internacional que mejore su situación, y Colombia y Chile no son las excepciones. Por esa razón, los libros blancos de 1997, 2002 y 2010 determinaron "la preservación de la 
nación, el mantenimiento de su independencia política, el mantenimiento de su soberanía y el mantenimiento de su integridad del territorio nacional" (Subsecretaría para las Fuerzas Armadas Gobierno de Chile, 2010). Así,

El régimen de la migración internacional constituye una arquitectura institucional y jurídica compleja, y en ocasiones fragmentada, de la cooperación internacional y el diálogo sobre cuestiones migratorias. De hecho, no existe un organismo o marco institucional único, unificado y global que tenga un mandato internacional en materia de migración internacional. (Naciones Unidas, 2015)

Por ello, los factores inestables deberán ser cambiados, dado que no se mantendrán a largo plazo para asegurar un desarrollo sustentable, es ahí donde el significado de las migraciones se vuelve tan importante debido a los ingresos que entran a los países de manos de las personas que se trasladan día a día a dichos lugares.

\section{Conclusión}

Un común denominador en el perfil de la política exterior de Brasil desde sus inicios como república (1822) es consolidar el Estado y la soberanía, pero estas variables no son suficientes por sí solas para lograr ser un Estado con proyección de poder y capacidad decisoria en el sistema internacional. Para que funcione la ecuación de esta política exterior ante el nuevo escenario de seguridad del mundo, es necesario que Brasil categorice las amenazas del COT y valorice las consecuencias de estas sobre la seguridad y defensa nacionales.

Una vez conjugadas estas variables, el Estado brasileño podrá planear, diseñar y ejecutar políticas públicas tendientes al alcanzar el interés nacional, de las que forma parte el código geopolítico local y regional, así como un cambio de doctrina, modernización, adaptación y transformación de sus Fuerzas Armadas. También es necesaria la participación de Brasil en subsistemas, para fortalecer la multilateralidad y poder así neutralizar al COT, mantener índices de crecimiento y desarrollo de su población, diezmar la corrupción de las instituciones estatales, proteger las fronteras, mantener la integridad de su infraestructura y el respeto a la dignidad humana y los valores democráticos. 
Es así como el espacio político, social y económico puede llegar a reducir las amenazas del COT.

Por otro lado, la política pública de Chile, en la lucha contra el crimen organizado, ha tenido grandes avances por parte del Estado, sin embargo, esto no ha sido suficiente, toda vez que debe haber un eje articulador entre la teoría y la comprobación empírica en los diferentes escenarios. Por ello, las políticas públicas son diversas y revisten una serie de factores estructurales que abarcan las relaciones bilaterales de los países, las leyes migratorias, las causas sociales, económicas, políticas y culturales, y la adaptabilidad e inserción en el país de acogida.

Chile ha ido aumentando progresivamente la cantidad de personas migrantes de diversas nacionalidades, cifra que se ha duplicado en un periodo de 10 años, cuya tendencia continúa en aumento. Expresiones xenófobas, discriminatorias y de estigmatización respecto de las personas extranjeras también han ido en aumento, a través de diferentes expresiones ciudadanas y también declaraciones de autoridades políticas. Esto revela que una modificación legal no es solamente necesaria, sino también urgente. En la actualidad la realidad está mucho más adelante que la legislación, y este escenario requiere contar con un marco legal que dé una adecuada protección a los derechos de las personas (Amnistía Internacional Chile, 2017).

Asimismo, el Estado chileno debe cambiar de punto de vista y ser flexible de manera gradual ante nuevos escenarios de migración, lo cual le permitiría entrar de nuevo a competir como lo hacía antes. Es fundamental enfatizar la diversificación de políticas públicas de los sectores sociales involucrados y así centrarse en una dinámica de crecimiento de mayor igualdad, en pro de la generación de empleo. De forma complementaria, se requiere apoyar la demanda regional y la expansión del consumo dentro de América Latina, con fundamento en un proceso de integración basado en la expansión de la dinámica para constituir equipo con las políticas públicas en contra del crimen organizado.

\section{Referencias}

Álvarez Calderón, C., y Rodríguez Beltrán, C. (2018). Ecosistemas criminales: hábitats para la convergencia y la globalización desviada. Revista Científica General José María Córdova, 16(24), 1-30. https://doi.org/10.21830/19006586.352 
Asamblea General de las Naciones Unidas. (1976). International Covenant on Economic, Social and Cultural Rights. https://www.ohchr.org/en/professionalinterest/pages/cescr.aspx

Ayoob, M. (1995). The third world security predicament: State making, regional conflict and the international system. Boulder/Lynne Rienner.

Banegas, A. (2017). ¿Existen estrategias para combatir las amenazas multidimensionales en la región?. Revista Politica y Estrategia, 129, 89-120.

Bartolomé, M. (2016). La seguridad internacional en el siglo XXI, más allá de Westfalia y Clausewitz. Ministerio de Defensa Nacional.

Bethell, L. (2009). Chile desde la independencia. Universidad Católica Cardenal Raúl Silva Henríquez.

Betts, A. (Ed.). (2011). Global migration governance. Oxford University Press.

Boneti, L. (2017). Políticas públicas por dentro. Clacso.

Brasil. (2012). Libro Blanco de Defensa Nacional. Ministerio de Defensa de Brasil. https://www. defesa.gov.br/arquivos/estado_e_defesa/livro_branco/lbdn_esp_net.pdf

Buzan, B., Waewer, O., y De Wilde, J. (1998). Security: A new framework for analysis. Lynne Rienner.

Casassas, J. (Coord.). (2005) La construcción del presente: el mundo desde 1848 hasta nuestros días. Ariel.

Cuervo-Ceballos, G. (2018). El crimen organizado transnacional como una amenaza híbrida para la Triple Frontera (Argentina, Paraguay y Brasil). Revista Cientifica General José María Córdova, 16(23), 43-61. http://dx.doi.org/10.21830/19006586.304

De Lucas, J. (1999). El desafío de las fronteras. Derechos humanos y xenofobia frente a una sociedad plural. Temas de Hoy.

Esquivel Triana, R. (junio de 2015). Intereses geopolíticos de Colombia. Estudios en Seguridady Defensa, 10(19), 71-86. https://www.esdeguerevistacientifica.edu.co/index.php/estudios/ article/view/69/46

García, I. (2013). El cambio climático. En cuadernos de estrategia. Instituto Español de Estudios Estratégicos \# 159 "los potenciadores del riesgo" (pp. 143-182). Edición Ministerio de Defensa de España. http://www.ieee.es/publicaciones-new/cuadernos-de-estrategia/2013/ Cuaderno_159.html

Gómez, J. A. (2010). La migración internacional: teorías y enfoques, una mirada actual. Semestre Económico, 13(26), 81-99.

González Martínez. M. A., Quintero Cordero, S. P. y Ripoll De Castro, A. (2018). La seguridad en la alianza del pacifico: ¿una plataforma para el estado colombiano? Revista Cientifica General José María Córdova, 16 (23), 63-73. https://doi.org/10.21830/19006586.102

Hobsbawn, E. (1998). Historia del siglo XX. Crítica.

Marín, E. (2016). Seguridad pública en Chile: percepción de criminalidad y violencia urbana: del hecho delictual a su percepción y representación social. En R. Lagos (Ed.), Seguridad Pública en Chile: del fenómeno global a la acción local. Ediciones Universidad Tecnológica Metropolitana. 
Mármora, L. (1987). Las políticas de migraciones internacionales: elementos para su definición. Revista del CIM, 5(7).

Medeiros, A. (2019). ¿¿Guerra al crimen organizado? Favelas e intervención militar. En C. Sampo, y S. Aldo (Comps.), La transformación de las Fuerzas Armadas en América Latina ante el crimen organizado. Ejército del Perú.

Ministerio de la Defensa Nacional de Chile. (2010). Libro de la Defensa Nacional de Chile.

Ministerio del Interior y Seguridad Pública. (2014). Plan Nacional de Seguridad Pública y Prevención de la Violencia y el Delito 2014-2018. Santiago de Chile: Subsecretaría de Prevención del Delito.

Naciones Unidas. (2015). Migración, derechos humanos y gobernanza: Manual para Parlamentarios. Unión Interparlamentaria; OIT; Naciones Unidas.

Nuechterlein, D. (1975). Los intereses nacionales de los Estados Unidos en un mundo cambiante. Roble S.A.

Organización Internacional para las Migraciones. (2017). Derecho internacional sobre migración. https://www.iom.int/es/derecho-internacional-sobre-migacion

Pardo, R. (1990). La política exterior del presidente César Gaviria Trujillo. Colombia Internacional, (12), 3-9.

Quintero Cordero, S. y Forero Garzón, M. (2018). Los libros blancos de defensa de Perú y Brasil: aspectos geopolíticos, seguridad regional e incidencia para Colombia. En C. Álvarez Calderón, y A. Fernández-Osorio (Eds.), Hacia una gran estrategia en Colombia: Construcción de politica pública en seguridad y defensa. Volumen 3: Pensamiento y cultura estratégica en Suramérica: un análisis de sus libros blancos de defensa (pp. 89-119). Sello Editorial ESMIC.

Quintero Cordero, S. y González Martínez, M. (2017). La evolución de la seguridad en el sistema internacional contemporáneo: un balance a partir de los casos de Estados Unidos y la Federación de Rusia. En A. Castillo-Castañeda, y C. Niño-González (Eds.), Nociones sobre seguridad y paz en las relaciones internacionales contemporáneas (pp. 447-471). Ediciones USTA.

Quintero Cordero, S. P. (2020). Seguridad ciudadana y participación de las comunidades en América Latina. Revista Cientifica General José María Córdova, 18(29), 5-24. https://doi. org/10.21830/19006586.561

Reynolds, P. (1977). Introducción al estudio de las relaciones internacionales. Editorial Tecnos.

Saín, M. (2002). Los dilemas de la reforma militar en la Argentina. Red de Seguridad y Defensa de América Latina (Resdal). https://www.resdal.org/Archivo/d000026e.htm

Sampo, C. y Aldo, S. (Comps.). (2019). La transformación de las Fuerzas Armadas en América Latina ante el crimen organizado. Ejército del Perú.

Sisco, C., y Chacón, O. (2004). Barry Buzan y la teoría de los complejos de seguridad. Revista Venezolana de Ciencia Política, 25, 125-146.

Villalpando, W. (2014). Crimen organizado transnacional. Caracteristicas de las redes delictivas internacionales. Astrea. 